\title{
Liquid crystals for photonics
}

\author{
Ignacio Moreno \\ Universidad Miguel Hernández \\ Departamento de Ciencia de Materiales \\ Óptica y Tecnología Electrónica \\ 03202 Elche, Spain \\ E-mail: i.moreno@umh.es
}

Liquid crystals (LCs) are organic materials that exhibit a state of matter whose properties lie between those of a conventional liquid and those of a solid crystal. An LC may flow like a liquid, but its molecules show a certain degree of ordering (positional and/or orientational), which gives them optical properties such as birefringence. In addition, they can be reoriented upon the application of a small external electric field. Therefore, they behave as reorientable birefringent materials, which can be employed to change the transmission, phase, or state of polarization of light. This is the basis of their great success as materials for the design of programmable optoelectronic components.

LC displays have come of age, having fulfilled their promise of several decades ago and having conquered the major market share in different flat electronic display application areas, such as television screens, laptop screens, screens in mobile phones, etc. However, their unique electrooptic properties make them also suitable for many other types of photonic applications. These include, for example, programmable optical filters and switches, beam-steering devices, integrated devices based on optical waveguiding, lasers, and optical nonlinear components. The remarkable advances in LC technology have led to the appearance of LC-based spatial light modulators (SLMs), with multiple uses as flat thin elements for the spatial amplitude, phase, or state of polarization modulation of a light beam. This special section of Optical Engineering is specifically devoted to all these other applications of LC materials.

Major research activity is being carried out in all basic, applied, and technological aspects of LC materials and its applications. I expect the following fields related to LCs to continue to be very active and relevant in the next few years:

- LC materials: including novel LC compounds and mixtures, LCs with nanoparticles (nanocomposites), and LCs with nonlinear optical effect, or with large birefringence.

- Modeling of LC components: including two- and threedimensional modeling of the optical and electrical properties of LCs in photonic crystals, optical fibers, and gratings.

- Manufacturing and technology of LC devices: including the alignment and addressing of LC devices, the implementation of LCs in waveguides, technological issues regarding new displays, and the manufacturing of LCs on silicon and switching components.

- Applications of LC devices: including lasing in liquid crystals, microwave applications, the use of LC devices for sensing, polarimetry and metrology, beam and pulse shaping based on LC-SLMs, and also biomedical and lighting/solar applications.

This special section of Optical Engineering presents an invited review paper (J. Beeckman, K. Neyts, and P. J. M. Vanbrabant) that reviews the state-of-the-art of the above mentioned topics, as well as extended contributions of selected communications that were mostly presented at the 3rd International Workshop on Liquid Crystals for Photonics - LCP2010, which was held in Elche, Spain, in September 2010. This third edition of the workshop was a continuation of the two previous editions, held in Gent, Belgium, in 2006 and in Cambridge, United Kingdom, in 2008. Next year, in December 2012, the workshop will take place for the first time outside of Europe, in Hong Kong, chaired by Professor V. G. Chigrinov. The contributions presented in this special section of Optical Engineering cover topics such as the development of new mixtures of LC materials to achieve high values of birefringence, the study and characterization of the electrooptical properties of LC materials cells, the application of finite element optical modeling for analyzing waveguides with LC material, as well as the application of electrical models for analyzing the LC modulators. The application of LC devices for color generation and the application of LC-SLMs in polarimetric imaging are topics also covered in this special section.

I would like to finish this guest editorial by expressing my sincere gratitude to all the authors who contributed to this special section, to the peer reviewers, who ensured high standards for the published papers, to the SPIE staff, especially to Karolyn Labes, for all her help along the entire process, and to the editor of Optical Engineering, Ron Driggers, for the opportunity to publish this special section.

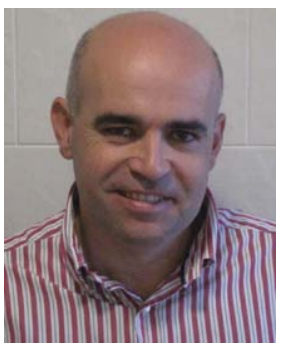

Ignacio Moreno completed his MS and $\mathrm{PhD}$ in physics in 1993 and 1996, respectively, at Universitat Autònoma de Barcelona, Spain. From 1996 to 1998 he was with the Department of Optics of Universidad de Valencia, and since 1998 he has belonged to the Department of Materials Science, Optics and Electronics Technology at Universidad Miguel Hernández in Elche. He has worked in a variety of topics related to liquid-crystal displays and their applications in diffractive and polarization optics. He has been a guest scientist at various research centers such as the Department of Physics at San Diego State University, the FEMTO Institut at the Université de FrancheComté, France, and the Departamento de Física at the Universidad de la Frontera, Chile. He is the editor of the Spanish journal Óptica Pura y Aplicada (OPA), edited by the Spanish Optical Society (SEDOPTICA). He is a member of SEDOPTICA, SPIE, and OSA. He is a senior member in both SPIE and OSA. He is currently a member of the SPIE Publications Committee. 